\title{
COMMUNICATION
}

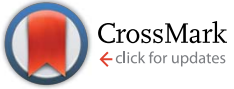

Cite this: RSC Adv., 2016, 6, 36085

Received 26th February 2016

Accepted 1st April 2016

DOI: $10.1039 / c 6 r a 05106 e$

www.rsc.org/advances

\section{Prediction of ionic liquid's heat capacity by means of their in silico principal properties $\dagger$}

\author{
Alessio Paternò, ${ }^{a}$ Roberto Fiorenza, ${ }^{a}$ Salvatore Marullo, ${ }^{b}$ Giuseppe Musumarra ${ }^{a}$ \\ and Salvatore Scirè ${ }^{\star a}$
}

The in silico principal properties (PPs) of ionic liquids (ILs), derived by means of the VolSurf+ approach, were used to develop a Partial Least Squares (PLS) model able to find a quantitative correlation among IL descriptors (accounting for both cationic and anionic structural features) and heat capacity values, providing affordable predictions validated by experimental $C_{p}$ measurements for an external set of ILs. In silico predictions allowed the selection of a limited number of structurally different ILs with similar $C_{\mathrm{p}}$ values, providing the possibility to select an optimal IL according to efficiency, as well as to environmental and economic sustainability. The present general procedure, using readily available descriptors for above 8000 ILs and adopting an accessible statistical procedure such as PLS, could be extended to other QSPR models.

\section{Introduction}

Ionic liquids (ILs) are a group of organic salts that have low melting points (usually defined as below $373 \mathrm{~K}$ ), formed of an organic cation and an inorganic or organic anion. ${ }^{1}$ In the last few decades ILs have attracted the attention of both the scientific and the industrial communities due to their high versatility, deriving from the large number of combinations of cations and anions which determines the properties of the resulting IL. This makes it possible to tune their physicochemical properties allowing the design and/or the synthesis of ILs for specific applications in various fields of technology, such as solvents, ${ }^{2,3}$ absorption media for gas separations, ${ }^{4,5}$ catalysts $^{6-8}$ heat transfer fluids and working fluids in electrochemical applications (batteries, solar cells). ${ }^{9-11}$

${ }^{a}$ Dipartimento di Scienze Chimiche, Università di Catania, Viale A. Doria 6, I-95125 Catania, Italy.E-mail: sscire@unict.it

${ }^{b}$ Dipartimento STEBICEF, Università di Palermo, Viale delle Scienze-Parco d'Orleans II, Ed. 17, 90128 Palermo, Italy

$\dagger$ Electronic supplementary information (ESI) available: Prediction of ionic liquids heat capacity by means of their in silico principal properties. See DOI: 10.1039/c6ra05106e
Heat capacity is a fundamental thermodynamic property of liquids needed to estimate heating and cooling requirements as well as heat-storage capacity of a material. Therefore it is an important parameter to verify the applicability of a liquid in heat-exchanger equipments. Heat-transfer fluids have a large number of industrial and consumer applications ranging from refrigeration at low temperatures to solar energy collection and storage at high temperatures. Heat capacities are also involved in the estimation of the temperature dependence of some thermodynamic properties of chemical compounds, such as entropy, enthalpy and Gibbs energy.

At present, the most exhaustive collection of IL heat capacities is the IL Thermo database, ${ }^{\mathbf{1 2}}$ containing available literature data. However for most ILs these values are still lacking. Moreover heat capacity data show a high degree of uncertainty, depending on the analytical method used, on the experimental apparatus precision and on the presence of impurities. Paulechka collected and critically reviewed experimental data on heat capacity of room-temperature ionic liquids in the liquid state (102 aprotic ILs from 63 literature references) ${ }^{13}$, pointing out that the purity of the ionic liquid can dramatically affect its properties. This has been specifically demonstrated for ILs containing water or halide impurities. ${ }^{\mathbf{1 4}}$

The huge number of combinations of cations and anions determine the physicochemical ILs properties whose knowledge is required to establish Quantitative Structure Property Relationships (QSPR) models aimed at an "intelligent" selection of ionic liquids for specific purposes.

In the present approach the prediction of IL heat capacity is based on in silico structural descriptors calculated adopting an approach called VolSurf $+{ }^{\mathbf{1 5 , 1 6}}$ using information coded into 3D GRID molecular interaction fields. ${ }^{17-20}$ The VolSurf+ procedure has been successfully applied to study the biological behaviour of organic molecules, e.g. structure-permeation relationships, ${ }^{\mathbf{1 5}}$ antitumour activities, ${ }^{21-23}$ phospholipidosis induction ${ }^{24}$ and recently to predict the aquatic toxicity of ionic liquids. ${ }^{25,26}$ VolSurf+ descriptors take into account several cationic IL structural features such as heterocyclic aromatic and non-aromatic cores, 
alkyl chain length, presence of oxygen atoms in the substituents as well as the properties of various inorganic and organic anions. Recently the VolSurf+ descriptors (128 for cations and 65 for anions) were compacted into a smaller number of IL descriptors, the so called Principal Properties (PPs, 5 for cations and 4 for anions). PPs, being orthogonal to each other, are highly informative variables for experimental design, maintaining most of the original matrix information, in this case the in silico properties for 218 heterocyclic cations and 38 organic and inorganic anionic counterparts of ionic liquids. ${ }^{27}$ IL PPs provided satisfactory QSPR models for IPC-81 cell line toxicity and acetylcholinesterase inhibition. ${ }^{27}$

Various approaches to computational chemistry used with ILs have been recently reviewed ${ }^{28}$ and the utility of combining computational and experimental approaches has been recognized. The above updated review confirms that the methodology adopted in the present communication was not previously applied in the ionic liquids field.

In the present work we intend to test the potentialities of the in silico ILs PPs to develop a Partial Least Squares (PLS) model able to quantitatively correlate the above descriptors to heat capacity to predict the heat capacity at constant pressure $\left(C_{\mathrm{p}}\right)$ for a large number of commonly used ILs with the aim to fulfil the requirements of the REACH (registration, evaluation, authorisation and restriction of chemicals) regulation by establishing priorities in planning the synthesis of new ILs for industrial applications.

\section{Results and discussion}

Heat capacity data, collected from the available literature, ${ }^{12,13}$ expressed as $\mathrm{J}\left(\mathrm{K}^{-1} \mathrm{~mol}^{-1}\right)$ were available for 65 ILs. These ILs (Table S1 $\dagger$ ) exhibit different structural features such as aromatic and non-aromatic heterocyclic cationic scaffolds (imidazoliums, pyridinium, piperidinium and pyrrolidinium), different length and presence of heteroatoms in the side chain as well as wide range of anions (halides, fluoborates, sulphonates and organic anions). The PLS analysis ${ }^{29}$ was carried out using a $65 \times$ 9 descriptor matrix (Table S1 $\dagger$ ) including 65 ILs and 9 variables (5 cation and 4 anion PPs $^{27}$ ) and the IL heat capacity as the $y$ response. The analysis provided an excellent 2 PLS orthogonal components model (see Table S2 $\dagger$ ) explaining $91.9 \%$ of $y$ variance $\left(Q^{2}=0.807\right)$ where the $1^{\text {st }}$ PLS component explains already $77.9 \%$ of $y$ variance $\left(Q^{2}=0.709\right)$.

In Fig. 1a the VIP (Variable Importance on the Projection) values bar plot is reported. VIP values give an indication (in absolute values) of what variables in the X block (PPs of ILs) are relevant to determine the dependent variable (ILs heat capacity). This plot shows that the response is affected by both cationic and anionic principal properties. In detail, the PLS loading weights plot (Fig. 1b) shows that the most important principal property is the anionic $\mathrm{PP}_{3^{-}}$which lies in the same quadrant and therefore contributes positively to $C_{\mathrm{p}}$ (i.e. the higher $\mathrm{PP}_{3^{-}}$, the higher $C_{\mathrm{p}}$ ) whereas cationic $\mathrm{PP}_{1^{+}}$, being in the opposite quadrant of the plot, provides a negative contribution. $\mathrm{PP}_{5}{ }^{+}$and $\mathrm{PP}_{4^{-}}$follow in order of importance. The physicochemical meaning of each single ILs PP has been
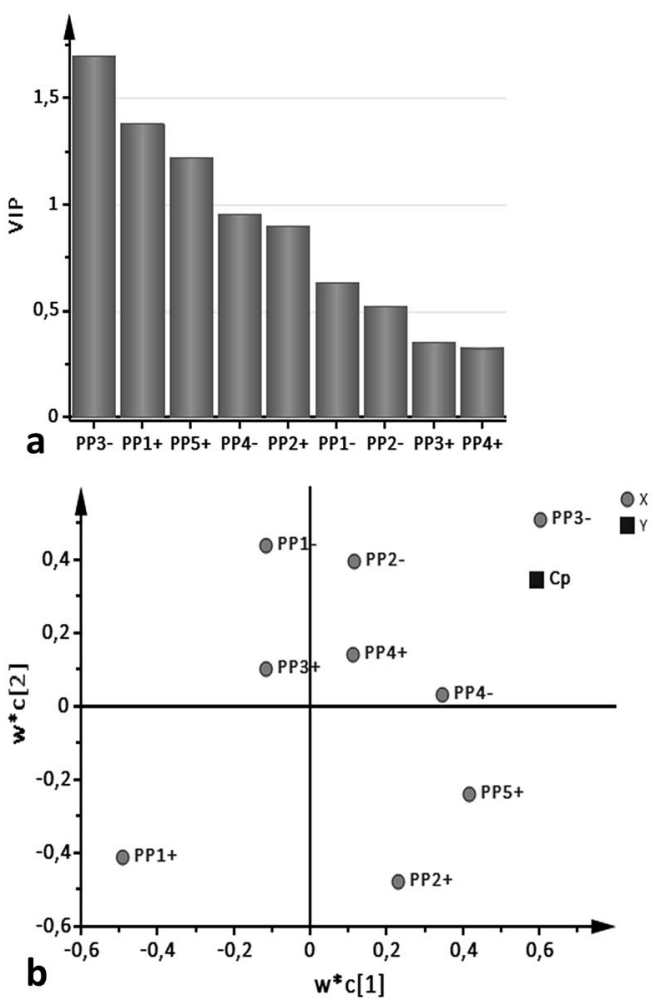

Fig. 1 (a) VIP values bar plot; (b) PLS loading weights plot.

commented. ${ }^{27}$ In the present case the $\mathrm{PP}_{1^{+}}$negative contribution means that a large cationic structure (wide surface, large volume and flexibility) together with hydrophobic surface and high molecular weight determine an increase in $C_{\mathrm{p}}$ values. On the other hand, a more water soluble cation is responsible for the decrease of $C_{\mathrm{p}}$. The cationic $\mathrm{PP}_{5^{+}}$refers to the H-bonding ability: a cation able to interact as donor contributes to increase the $C_{\mathrm{p}}$ value, and vice versa. Anion PPs have a high effect on the response, in particular anionic $\mathrm{PP}_{3^{-}}$and $\mathrm{PP}_{4^{-}}$, both positively contributing to the $C_{\mathrm{p}}$ value. Consequently large surface and volume, high hydrophilicity and polarizability (related to high positive $\mathrm{PP}_{3}$-values) and the unbalance between hydrophobic areas and the anions barycentre (referring to positive $\mathrm{PP}_{4^{-}}$), contribute to the increase of $C_{\mathrm{p}}$. On the other hand, anions with strong $\mathrm{H}$-bonding ability (high negative $\mathrm{PP}_{3}$ ) result in lower $C_{\mathrm{p}}$ values.

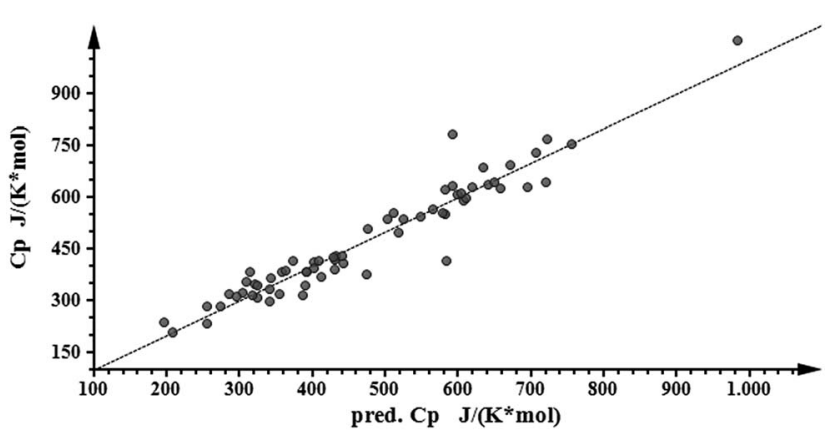

Fig. 2 Predicted vs. experimental literature data. 


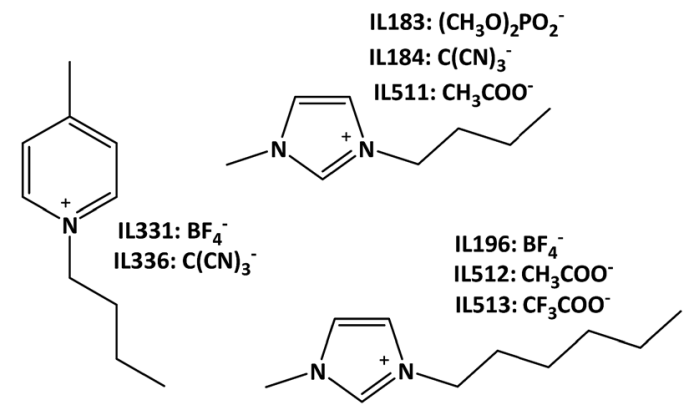

Scheme 1 ILs structures used as validation set.

It is worth mentioning that the present QSPR approach is able to provide an estimate of the importance and an interpretation of physico-chemical parameters in relation to $C_{\mathrm{p}}$ considering simultaneous variations in both the cation (heterocyclic core, side chain length, presence of oxygen atoms in the side chain) and the anion structural features.

The plot of predicted $v s$. experimental literature data is reported in Fig. 2 (see also Table S1 $\dagger$ ) where a good agreement between experimental and predicted values can be observed. This correlation can be considered as very good, taking into account the intrinsic variability of heat capacity data reported in the literature, strongly affected by errors due to different apparatus and analytical methods used in the $C_{\mathrm{p}}$ determination and to the presence of impurity in the ILs. Modelling of a series of ILs derivative thermodynamic properties including heat capacity by means of ePC-SAFT theoretical model has recently been reported ${ }^{30}$ and percentage prediction errors lower than $20 \%$ were claimed to be much better than those previously reported in the literature. The correlation reported in Fig. 2, including a much higher number of ILs with four aromatic and non-aromatic cationic heterocyclic cores and a wide variety of inorganic and organic anions, points out a significant improvement of the PLS model prediction ability.

In order to validate the above model an external set of 7 ILs (Scheme 1) was used, and $C_{\mathrm{p}}$ values were measured experimentally by Differential Scanning Calorimetry (DSC, see also ESI $\dagger$ ). Table 1 points out a good agreement between

Table 1 Experimental and predicted $C_{p}$ values for test set ILS

\begin{tabular}{llll}
\hline & $\begin{array}{l}C_{\mathrm{p}}(\text { liter. })^{a} \\
\text { Entry }\end{array}$ & $\begin{array}{l}C_{\mathrm{p}}(\mathrm{exp} .)^{b} \\
\mathrm{~J}\left(\mathrm{~K}^{-1} \mathrm{~mol}^{-1}\right)\end{array}$ & $\begin{array}{l}C_{\mathrm{p}}(\mathrm{pred} .) \\
\mathrm{J}\left(\mathrm{K}^{-1} \mathrm{~mol}^{-1}\right)\end{array}$ \\
\hline 183 & & 437.4 & 459.1 \\
184 & & 370.5 & 413.0 \\
196 & 429.8 & 452.0 & 440.8 \\
$331^{c}$ & 414.0 & 424.1 & 409.0 \\
336 & & 407.6 & 409.2 \\
511 & 383.2 & 360.0 & 392.3 \\
512 & & 400.1 & 420.3 \\
513 & & 481.0 & 470.6
\end{tabular}

${ }^{a}$ From ref. 13. ${ }^{b}$ This work. ${ }^{c}$ The $C_{\mathrm{p}}$ value for this learning set IL was measured to test the reliability of the adopted experimental procedure.
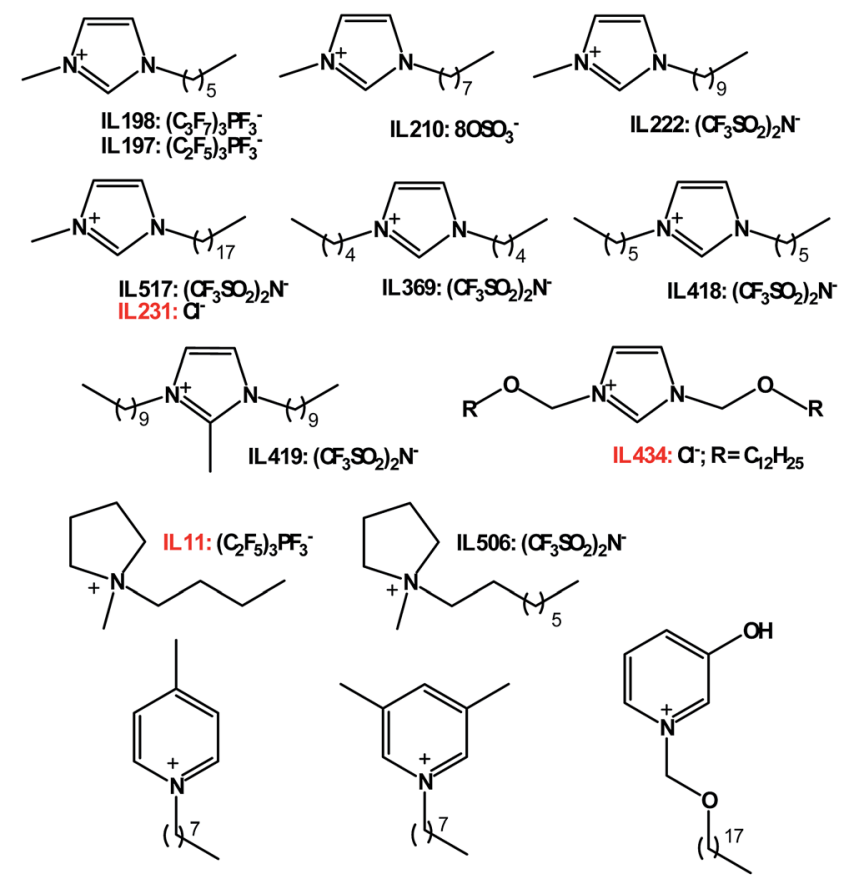

IL503: $\left(\mathrm{CF}_{3} \mathrm{SO}_{2}\right)_{2} \mathrm{~N}^{-}$<smiles></smiles><smiles>C[14C](=O)OC[n+]1cccc(O)c1</smiles>

Scheme 2 Ionic liquids with predicted $C_{\mathrm{p}}>700 \mathrm{~J}\left(\mathrm{~K}^{-1} \mathrm{~mol}^{-1}\right)$. ILs with DModX higher than 1.60 in red.

experimental and predicted $C_{\mathrm{p}}$ values for the test set of the 7 ILs, thus confirming that the PLS approach represents a valid tool to predict heat capacity starting from PP in silico structural descriptors.

In PLS $Q^{2}$ estimates the predictive power of the model, however a further internal validation tool, the so-called response permutation testing, ${ }^{31}$ can be adopted to assess the statistical significance of the predictions. The resulting permutation plot (Fig. S1 in ESI $\dagger$ ) clearly excludes fitting of random response data. ${ }^{31}$

The reliability of the PLS model suggested that predictions could be extended to a higher number of commonly used ILs (the same set of 520 arbitrarily chosen in ref. 27) also reported in Table S1. $\dagger$ However, the prediction ability for a high number of ILs is different for each single IL. Guidance on the "goodness" of the prediction can be evaluated by DModX values, the model residuals in the $X$ matrix, which give an estimate of the similarity of a new observation to the training set observation. Predictions for ILs exhibiting DModX values higher than 1.60 in Table S1 $\uparrow$ should be regarded with caution, ${ }^{32}$ the higher DModX the lower the prediction reliability.

In Fig. 2 a single IL (IL 517, 1-methyl-3-octadecylimidazolium 1,1,1-trifluoro- $N$-[(trifluoromethyl)sulfonyl] methanesulfonamide) exhibits an extraordinary high experimental $C_{\mathrm{p}}$ value, above $1000 \mathrm{~J}$ $\left(\mathrm{K}^{-1} \mathrm{~mol}^{-1}\right)$, in agreement with the PLS model prediction, while very few ILs can be found in the range 700-1000 $\left.\mathrm{J}_{(\mathrm{K}}^{-1} \mathrm{~mol}^{-1}\right)$. Predictions in Table $\mathbf{S 1 \dagger}$ for as many as 16 ILs lie in the above range, in particular IL 419 (1,3-didecyl-2-methylimidazolium 1,1,1trifluoro- $N$-[(trifluoromethyl)sulfonyl] methanesulfonamide) exhibits a predicted $C_{\mathrm{p}}$ value comparable with that of IL 517. It is 
worth mentioning that this set includes ILs with different aromatic (imidazolium, pyridinium) and non-aromatic (pyrrolidinium) cationic scaffolds, side chains containing oxygen atoms, chlorides, sulfonates and sulfates, fluorine organic anions etc. (Scheme 2). Table S1† provides in silico opportunities for the selection of a limited number of ILs with comparable heat capacity efficiency. ILs reported in Scheme 2 can be considered as alternative candidates for experimental $C_{\mathrm{p}}$ measurements when a high $C_{\mathrm{p}}$ value is desired.

Those exhibiting in Table S1† DModX values lower than 1.60 should be preferred to those (indicated in red in Scheme 2) with a lower prediction reliability.

\section{Experimental}

\section{Computational methods}

The partial least squares projections to latent structures (PLS) ${ }^{29}$ chemometric tool, available in the SIMCA Software package (SIMCA 13.0.3), ${ }^{31}$ was adopted. Details on the PLS procedure are reported in ESI. $\dagger$

\section{Test set ILs structures}

The structures of test set ILs used for experimental determinations of heat capacities are reported in Scheme 1. Some of them were purchased (ILs 183, 184 and 336), the others synthesized in our laboratory (ILs 196, 331, 512, 513). Experimental details are reported in ESI. $\dagger$

\section{Heat capacity measurements}

The heat capacities were measured over the temperature ranging from 303 to $353 \mathrm{~K}$ at atmospheric pressure condition by means of DSC analysis. Water was used as standard to check the heat capacity measurements according to the method reported by Chiu et $a l .^{33}$ Experimental details are reported as ESI. $\dagger$

\section{Conclusions}

A PLS model was able to correlate principal properties of cationic and anionic counterparts of $\mathrm{ILs}^{27}$ with heat capacity and to predict $C_{\mathrm{p}}$ values for a large number of ILs. In silico predictions may lead to the selection of a limited number of structurally different ILs for industrial experimental design requiring high $C_{\mathrm{p}}$ values, providing the possibility to select an optimal IL according not only to an efficiency criterion but also to synthetic affordability, environmental sustainability and economic considerations. The present procedure, using readily available descriptors for above 8000 ILs and adopting an accessible statistical procedure such as PLS, could be easily extended to the development of QSPR models for the prediction of other ILs physico-chemical properties providing new tools to the wide scientific community interested in the design of ILs for specific industrial applications.

\section{Acknowledgements}

We thank the University of Catania for partial financial support (FIR project ECDF5E) and for a PhD grant (to AP).

\section{References}

1 R. Hayes, G. G. Warr and R. Atkin, Chem. Rev., 2015, 115, 6357-6426.

2 J. Scholz, V. Hager, X. Wang, F. T. U. Kohler, M. Sternberg, M. Haumann, N. Szesni, K. Meyer and P. Wasserscheid, ChemCatChem, 2014, 6, 162-169.

3 E. E. L. Tanner, R. R. Hawker, H. M. Yau, A. K. Croft and J. B. Harper, Org. Biomol. Chem., 2013, 11, 7516-7521.

4 S. Rodríguez-Sánchez, P. Galindo-Iranzo, A. C. Soria, M. L. Sanz, J. E. Quintanilla-López and R. Lebrón-Aguilar, J. Chromatogr. A, 2014, 1326, 96-102.

5 M. D. Joshi and J. L. Anderson, RSC Adv., 2012, 2, 5470-5484. 6 H. Olivier-Bourbigou, L. Magna and D. Morvan, Appl. Catal., A, 2010, 373, 1-56.

7 C. S. Consorti, G. L. P. Aydos and J. Dupont, Chem. Commun., 2010, 46, 9058-9060.

8 F. D'Anna, S. Marullo, P. Vitale and R. Noto, Eur. J. Org. Chem., 2011, 5681-5689.

9 S. Fletcher, F. Sillars, N. Hudson and P. Hall, J. Chem. Eng. Data, 2010, 55, 778-782.

10 A. N. Soriano, B. T. Doma Jr and M. H. Li, J. Chem. Thermodyn., 2008, 40, 1654-1660.

11 J. M. P. França, C. A. Nieto de Castro, M. M. Lopes and V. M. B. Nunes, J. Chem. Eng. Data, 2009, 54, 2569-2575.

12 IL Thermo Database, http://www.nist.gov/srd/nist147.htm, accessed 1 October 2015, NIST Standard Reference Database Number 147, National Institute of Standards and Technology, Gaithersburg, MD.

13 Y. U. Paulechka, J. Phys. Chem. Ref. Data, 2010, 39(3), 033108.

14 K. R. Seddon, A. Stark and M. J. Torres, Pure Appl. Chem., 2000, 72, 2275-2287.

15 G. Cruciani, P. Crivori, P. A. Carrupt and B. Testa, J. Mol. Struct.: THEOCHEM, 2000, 503, 17-30.

16 VolSurf + Manual, http://www.moldiscovery.com/docs/ vsplus/.

17 E. Carosati, S. Sciabola and G. Cruciani, J. Med. Chem., 2004, 47, 5114-5125.

18 P. J. Goodford, J. Med. Chem., 1985, 28, 849-857.

19 D. N. A. Boobbyer, P. J. Goodford, P. M. Mcwhinnie and R. C. Wade, J. Med. Chem., 1989, 32, 1083-1094.

20 R. Wade, K. J. Clerk and P. J. Goodford, J. Med. Chem., 1993, 36, 140-147.

21 V. Barresi, C. Bonaccorso, G. Consiglio, L. Goracci, N. Musso, G. Musumarra, C. Satriano and C. G. Fortuna, Mol. BioSyst., 2013, 9, 2426-2429.

22 C. G. Fortuna, V. Barresi, C. Bonaccorso, G. Consiglio, S. Failla, N. Musso and G. Musumarra, Eur. J. Med. Chem., 2012, 47, 221-227.

23 C. G. Fortuna, V. Barresi and G. Musumarra, Bioorg. Med. Chem., 2010, 18, 4516-4523. 
24 L. Goracci, S. Buratta, L. Urbanelli, G. Ferrara, R. Di Guida, C. Emiliani and S. Cross, Eur. J. Med. Chem., 2015, 92, 49-63.

25 A. Paternò, F. D'Anna, G. Musumarra, R. Noto and S. Scirè, RSC Adv., 2014, 4, 23985-24000.

26 A. Paternò, G. Bocci, L. Goracci, G. Musumarra and S. Scirè, SAR QSAR Environ. Res., 2016, 27, 1-15.

27 A. Paternò, G. Bocci, G. Cruciani, C. G. Fortuna, L. Goracci, S. Scirè and G. Musumarra, SAR QSAR Environ. Res., 2016, 27, 221-244.

28 S. T. Keaveney, J. B. Harper and A. K. Croft, RSC Adv., 2015, 5, 35709-35729.
29 S. Wold, M. Sjostrom and L. Eriksson, PLS in Chemistry, in The Encyclopedia of Computational Chemistry, ed. PvR Schleyer, John Wiley \& Sons, Chichester, 1998, pp. 20062020.

30 G. Shen, C. Held, X. Lu and X. Ji, Fluid Phase Equilib., 2015, 405, 73-82.

31 SIMCA 13, Software from MKS Umetrics AB, Malmo, Sweden. 32 S. Wold and M. Sjöström, Chemometrics: Theory and Application, ACS Symposium Series, ed. B. R. Kowalski, Washington, 1977, p. 243.

33 L.-F. Chiu, H.-F. Liu and M.-H. Li, J. Chem. Eng. Data, 1999, 44, 631-636. 